\title{
Cavity quantum electro-dynamics with solid-state emitters in aperiodic nano-photonic spiral devices
}

Oliver J. Trojak, ${ }^{1, *}$ Sean Gorsky, ${ }^{2, *}$ Fabrizio Sgrignuoli, ${ }^{2}$ Felipe A. Pinheiro, ${ }^{3}$ Suk-In Park, ${ }^{4}$ Jin Dong Song, ${ }^{4}$ Luca Dal Negro, $, 2,5,6, \dagger$ and Luca Sapienza ${ }^{1, \ddagger}$

${ }^{1}$ Department of Physics and Astronomy, University of Southampton, Southampton SO17 1BJ, United Kingdom

${ }^{2}$ Department of Electrical \& Computer Engineering and Photonics Center,

Boston University, 8 Saint Mary's St., Boston, Massachusetts, 02215, USA.

${ }^{3}$ Instituto de Fisica, Universidade Federal do Rio de Janeiro, Rio de Janeiro-RJ 21941-972, Brazil

${ }^{4}$ Center for Opto-Electronic Materials and Devices Research,

Korea Institute of Science and Technology, Seoul 136-791, South Korea

${ }^{5}$ Division of Material Science \& Engineering, Boston University,

15 Saint Mary's St. Brookline, Massachusetts, 02446, USA

${ }^{6}$ Department of Physics, Boston University,

590 Commonwealth Ave., Boston, Massachusetts, 02215, USA 


\begin{abstract}
Integrated quantum devices are at the basis of the realisation of scalable, high-performance quantum technology, including quantum computers and quantum communication schemes, where single photons are emitted, guided, manipulated and detected on a chip. Engineered nano-devices enable the efficient confinement of light and, ultimately, the control of the spontaneous emission dynamics of single emitters, which is crucial for cavity quantum electrodynamics experiments and for the development of classical and quantum light sources. Here, we report on the demonstration of enhanced light-matter interaction and Purcell effects on a chip, based on bio-inspired aperiodic devices fabricated in gallium arsenide. Indium arsenide single quantum dots are used as internal light sources to image, by means of micro-photoluminescence spectroscopy, the optical modes supported by photonic membranes with Vogel-spiral geometry. These emitters are also used to probe the density of optical states, modified by the aperiodic devices, by means of time-resolved spectroscopy. Our results show cavity quantum electrodynamics effects providing strong modifications of the spontaneous emission decay of single optical transitions. In particular, thanks to the significant modification of the density of optical states demonstrated in Vogel-spiral photonic structures, we show control of the decay lifetime of single emitters with a dynamic range reaching 20, thus opening the path to the implementation of aperiodic geometries in active classical and quantum devices.
\end{abstract}


Integrated photonic devices are at the basis of all-optical chips, essential ingredients for quantum information technology applications ${ }^{1}$. In such devices, one of the key features relies in the control of the emission properties of integrated solid-state quantum emitters, most importantly the spontaneous emission dynamics ${ }^{2,3}$. In this way, one is able to control, for instance, the singlephoton emission repetition rate and improve the coherence of the emitted quantum light, by reducing the spontaneous emission lifetime ${ }^{4}$. To this end, several photonic geometries have been developed, including photonic crystal cavities ${ }^{5}$ and waveguides ${ }^{6}$, micropillar ${ }^{7}$ and circular grating cavities ${ }^{8}$, and disordered photonic devices ${ }^{9}$. In this work, we put forward a platform, based on aperiodic geometries, that provides enhanced light-matter interaction, compatible with III-V semiconductor technology, requiring less stringent fabrication accuracies, and allowing control over spontaneous emission dynamics of quantum emitters and optical angular momentum of the emitted light.

We demonstrate the potential of nano-photonic devices characterised by a bio-inspired deterministic aperiodic structure, based on Vogel-spiral geometries (see Fig. 1a and Supplementary Material for more details), as an on-chip platform for cavity quantum electrodynamics experiments. Aperiodic order, in particular following spiral configurations, is present in natural systems where, for instance, the arrangements of leaves and seeds in plants follows Fibonacci series. Such findings are at the basis of the fascinating area of phyllotaxis ${ }^{10}$ and aperiodic arrangements in photonic devices have shown to provide unique properties, including anomalous transport and light localisation ${ }^{11}$, with applications in optical sensing, light emission and lasing, photo-detection, optical imaging and non-linear optics ${ }^{12}$. The study of bio-inspired systems has attracted considerable interest in classical photonics ${ }^{13}$ and we here implement such an approach to quantum photonics.

We experimentally investigate, by means of micro-photoluminescence spectroscopy, the optical properties of gallium arsenide (GaAs) membranes confining light in the near infra-red, where air holes are arranged in a golden-angle Vogel-spiral geometry (see Fig. 1a). The devices were designed by considering the local density of optical states (LDOS) that describes the radiation dynamics of a source embedded into an arbitrary structure. Our simulations are based on multi-polar expansions, used to evaluate the two-dimensional electromagnetic Green's tensor, from which the LDOS is calculated (for more details, see Supplementary Material). The LDOS is evaluated for arbitrary arrays of parallel and non-overlapping circular cylinders embedded in a non-absorbing medium. The analysis is limited to the transverse electric polarization, since this is the polarization for which a band-gap is expected to occur ${ }^{14}$. An effective refractive index is introduced in order 
to take into account out-of-plane losses, so that the dispersion of the dielectric material is replaced by the effective index of the fundamental guided mode in the three-dimensional heterostructure without air-holes ${ }^{15}$ (see Supplementary Material for more details). Fig. 1(a) shows a map of the LDOS as a function of wavelength and air-hole diameters, and (b) depicts one-dimensional cuts of the LDOS map. Photonic band-gap and pseudo-gaps (secondary gaps of smaller amplitudes) can be seen as yellow bands in Fig. 1(a) and their behaviour is determined by the unique multifractal structural properties of the golden-angle Vogel spiral ${ }^{16}$. Several peaks, more clearly visible in Fig. 1(b), correspond to long-lived modes generated by the first-neighbour distributions of the spiral elements ${ }^{11}$. Localized band-edge modes are formed when ring-shaped regions of similar inter-particle separation $d$ are placed between two regions with different values of $d$, creating a photonic heterostructure ${ }^{16}$. Such band-edge modes are typically spatially extended, long-lived ${ }^{11}$, and less sensitive to local perturbations: golden-angle Vogel spirals are therefore more robust to fabrication imperfections than traditional photonic crystals ${ }^{17}$. The existence of isotropic gaps also implies reductions in the group velocity of propagating light, thus increasing the light-matter interaction. Such properties are particularly suitable for non-linear optics applications and for the realisation of low-threshold lasers ${ }^{14,17}$. The LDOS was evaluated at the center of a spiral composed by 350 air-holes and the optimised design resulted in an average hole separation of $220 \mathrm{~nm}$.

We use this aperiodic platform to carry out quantum electro-dynamics experiments by coupling single emitters to the confined optical modes. To this end, we have fabricated golden-angle Vogelspiral membranes in GaAs, as shown in Fig. 2a, containing a single layer of InAs quantum dots, grown via Stranski-Krastanov technique (for more details about the fabrication process, see the Supplementary Material). We show that the emission dynamics of single emitters can be strongly modified due to the modulation of the density of optical states in the aperiodic spiral device (see Supplementary Material for more details on LDOS simulations).

We excite quantum dot emitters, cooled down to cryogenic temperatures, with an above-band 455-nm light-emitting diode and image the confined optical modes on an electron-multiplied charge-coupled device (see Fig. 2b). In Fig. 3, examples of photo-luminescence images of the optical modes confined by aperiodic photonic spiral devices are shown: extended annular photonic modes are visible, in accordance with the confined optical modes predicted by the simulations in panel (a) (see Supplementary Material for more details on the calculations). Thanks to our imaging technique $^{18}$, we directly visualise the confined optical modes and, therefore, we are able to locate the maximum intensity of the confined electromagnetic fields. As shown in the images in Fig. 3b- 
g, different devices support confined optical modes with different spatial distributions, showing the versatility of the aperiodic geometries under study. Furthermore, this imaging technique could be used to selectively deposit single emitters at specific locations on the devices in order to enhance the light-matter interaction. If such an approach is followed, care should be taken in the polarisation of the emitter, given that the aperiodic devices under study are effective for transverse electric polarised light, as shown by numerical simulations.

The InAs quantum dots embedded within the GaAs suspended membranes are then excited with a picosecond-pulsed $785 \mathrm{~nm}$ diode laser to carry out time-resolved photo-luminescence spectroscopy. The laser excitation power is chosen so as to be below the saturation level of single quantum dot emission lines. Examples of photo-luminescence spectra are shown in Fig. 4 (left panels) where sharp peaks that are spectrally resolution-limited, signature of atom-like transitions in single quantum dots, are visible. Single emission lines are then spectrally filtered (see Fig. 2b) with sub-nanometer accuracy, so as to select a single emission line, corresponding to a two-level transition within a selected quantum dot, to be sent to a silicon avalanche photo-diode for photon counting: time-resolved measurements of the emitted intensity are carried out and the experimental results are fitted with exponential decays to extract the lifetimes of the transitions. Exponential functions as expected from the spontaneous emission decay dynamics of single quantum dot, consistent with previous reports ${ }^{4,7,9}$. When evaluating the decay dynamics of the intensity of the emitted light, we observe strong modifications of the spontaneous emission rate. InAs/GaAs quantum dots located in an unpatterned region of the membrane show lifetimes of $\sim 1 \mathrm{~ns}$. When coupled to the confined optical modes in our aperiodic photonic devices, instead, we measure lifetimes as short as $\sim 500 \mathrm{ps}$ : the ratio between the lifetime of quantum dots outside the photonic device and the one measured within the aperiodic structure shows that the spontaneous emission dynamics is Purcell enhanced by a factor 2 . We also measure lifetimes of quantum dots within the aperiodic devices as long as $10 \mathrm{~ns}$, showing a slow-down of the decay dynamics of a factor 10 (see Fig. 4, right panels). Considering the ratio between the longest and shortest lifetime measured, we show a modification of the spontaneous emission rate of single quantum dots with a dynamic range of 20. Such strong changes in the emission lifetimes reflect the modified density of optical states due to the presence of multifractal band-gap and pseudo-gaps in bio-inspired Vogel-spiral structures ${ }^{16}$. Our calculations of the Purcell enhancement show that, for optimally positioned emitters ${ }^{8,19,20}$, Purcell enhancements up to two-orders of magnitude are theoretically expected, showing the potential of aperiodic devices for efficient lifetime control. Another possible source of reduction 
in the measured enhancements with respect to the simulated values can be related to imperfections present in the fabricated device that do not allow to obtain the optimal confinement of the electromagnetic field, due to scattering processes that act as losses in the system.

In conclusion, our results show that aperiodic nano-photonic devices based on bio-inspired aperiodic geometry are an effective platform for for cavity quantum electrodynamics experiments with integrated quantum emitters in the near-infrared range of wavelengths. We foresee the application of aperiodic geometries for integrated quantum photonics, nano-lasers, optical sensors and nonlinear optics ${ }^{21}$, and the development of active devices operating from the visible to the telecommunication range of wavelengths. In particular, the light confinement and the Purcell enhancement that we have here demonstrated could be utilized for the development of laser and single-photon devices where optical angular momentum, supported by such aperiodic geometries ${ }^{22}$, is imparted to the emitted radiation and used as an extra degree of freedom to encode information for quantum information technology applications ${ }^{23}$.

\section{Supplementary Material}

Supplementary material provides more in-depth discussion of the device geometry, fabrication details and simulations.

\section{Data Availability Statement}

The data that support the findings of this study are available from the corresponding author upon reasonable request.

\section{Author contributions}

LS conceived the optical set-up and built it together with OJT. OJT fabricated the devices, carried out the experiments and analysed the data, together with LS. SIP and JDS grew the quantum dot material. LS, FAP, LDN conceived the research activities and discussed the results, together with the other authors. LS supervised the experimental part of the project and wrote the manuscript with contributions from the other authors. LDN supervised the design and modeling contributions. SG developed the numerical tools utilized in the paper. SG and FS performed, analyzed, and or- 
ganized the simulation results. SG wrote the Supplementary Informations with the help of FS and LDN.

\section{Acknowledgments}

FAP acknowledges financial support from CNPq, CAPES, and FAPERJ. JDS acknowledges support from IITP grant funded by the Korea government (MSIT No. 20190004340011001). LDN acknowledges partial support from the Army Research Laboratory under Cooperative Agreement Number W911NF-12-2-0023 for the development of theoretical methods utilized in the paper. LS acknowledges partial support from the Royal Society, grant RG170217, the Leverhulme Trust, grant IAF-2019-013, EPSRC, grant EP/P001343/1.

* These authors contributed equally to this work

$\dagger$ Electronic address: dalnegro@bu.edu

\# Electronic address: 1.sapienza@soton.ac.uk

1 J. O’Brien, A. Furusawa, J. Vuckovic, Photonic quantum technologies, Nature Photonics 3, 687 (2009).

2 P. Ginzburg, Cavity quantum electrodynamics in application to plasmonics and metamaterials, Reviews in Physics 1, 120 (2016).

3 P. Ginzburg, D.J. Roth, M.E. Nasir, P. Segovia, A.V. Krasavin, J. Levitt, L.M. Hirvonen, B. Wells, K. Suhling, D. Richards et al., Spontaneous emission in non-local materials, Light: Science \& Applications 6, e16273 (2017).

4 S. Ates, S.M. Ulrich, S. Reitzenstein, A. Loffler, A. Forchel, P. Michler, Post-selected indistinguishable photons from the resonance fluorescence of a single quantum dot in a microcavity, Physical Review Letters 103, 167402 (2009).

5 T. Asano, Y. Ochi, Y. Takahashi, K. Kishimoto, S. Noda, Photonic crystal nanocavity with a Q factor exceeding eleven million, Optics Express 25, 1769 (2017).

${ }^{6}$ H. Thyrrestrup, L. Sapienza, P. Lodahl, Extraction of the beta-factor for single quantum dots coupled to a photonic crystal waveguide, Applied Physics Letters 96, 231106 (2010).

7 N. Somaschi, V. Giesz, L. De Santis, J.C. Loredo, M.P. Almeida, G. Hornecker, S.L. Portalupi, T. Grange, C. Antn, J. Demory et al., Near-optimal single-photon sources in the solid state, Nature Photon- 
ics 10, 340 (2016).

8 L. Sapienza, M. Davanco, A. Badolato, K. Srinivasan, Nanoscale optical positioning of single quantum dots for bright and pure single-photon emission, Nature Communications 6, 7833 (2015).

9 L. Sapienza, H. Thyrrestrup, S. Stobbe, P.D. Garcia. S. Smolka, P. Lodahl, Cavity quantum electrodynamics with Anderson-localized modes, Science 327, 1352 (2010).

10 G.J. Mitchison, Phyllotaxis and the Fibonacci series, Science 196, 270 (1977).

11 F. Sgrignuoli, R. Wang, F.A. Pinheiro, L. Dal Negro, Localization of scattering resonances in aperiodic Vogel spirals, Physical Review B 99, 104202 (2019).

12 L. Dal Negro, R. Wang, F. Pinheiro, Structural and spectral properties of deterministic aperiodic optical structures, Crystals 6, 161 (2016).

13 S. Tadepalli, J.M. Slocik, M.K. Gupta, R.R. Naik, S. Singamaneni, Bio-optics and bio-inspired optical materials, Chemical Reviews 117, 12705 (2017).

14 M. Soljačić, J.D. Joannopoulos, Enhancement of nonlinear effects using photonic crystals, Nature Materials 3, 211 (2004).

15 M. Qiu, Effective index method for heterostructure-slab-waveguide-based two-dimensional photonic crystals, Applied Physics Letters 81, 1163 (2002).

16 J. Trevino, S.F. Liew, H. Noh, H. Cao, L. Dal Negro, Geometrical structure, multifractal spectra and localized optical modes of aperiodic Vogel spirals, Optics Express 20, 3015 (2012).

17 M.E. Pollard, G.J. Parker, Low-contrast bandgaps of a planar parabolic spiral lattice, Optics Letters 34, 2805 (2009).

18 T. Crane, O.J. Trojak, J.P. Vasco, S. Hughes, L. Sapienza, Anderson localisation of visible light on a nanophotonic chip, ACS Photonics 4, 2274 (2017).

19 J. Liu, M. Davanco, L. Sapienza, K. Konthasinghe, J.V. De Miranda Cardoso, J.D. Song, A. Badolato, K. Srinivasan, Cryogenic photoluminescence imaging system for nanoscale positioning of single quantum emitters, Review of Scientific Instruments 88, 023116 (2017).

20 M. Gschrey, F. Gericke, A. Schuessler, R. Schmidt, J. H. Schulze, T. Heindel, S. Rodt, A. Strittmatter, and S. Reitzenstein, In situ electron-beam lithography of deterministic single-quantum dot mesastructures using low-temperature cathodoluminescence spectroscopy, Appl. Phys. Lett. 102 (2013).

21 L. Dal Negro, S.V. Boriskina, Deterministic aperiodic nanostructures for photonics and plasmonics applications, Laser \& Photonics Reviews 6, 178 (2012).

22 L. Dal Negro, N. Lawrence, J. Trevino, Analytical light scattering and orbital angular momentum spectra 
of arbitrary Vogel spirals, Optics Express 20, 18209 (2012).

23 G. Molina-Terriza, J.P. Torres, L. Torner, Twisted Photons, Nature Physics 3, 305 (2007). 
Figures 

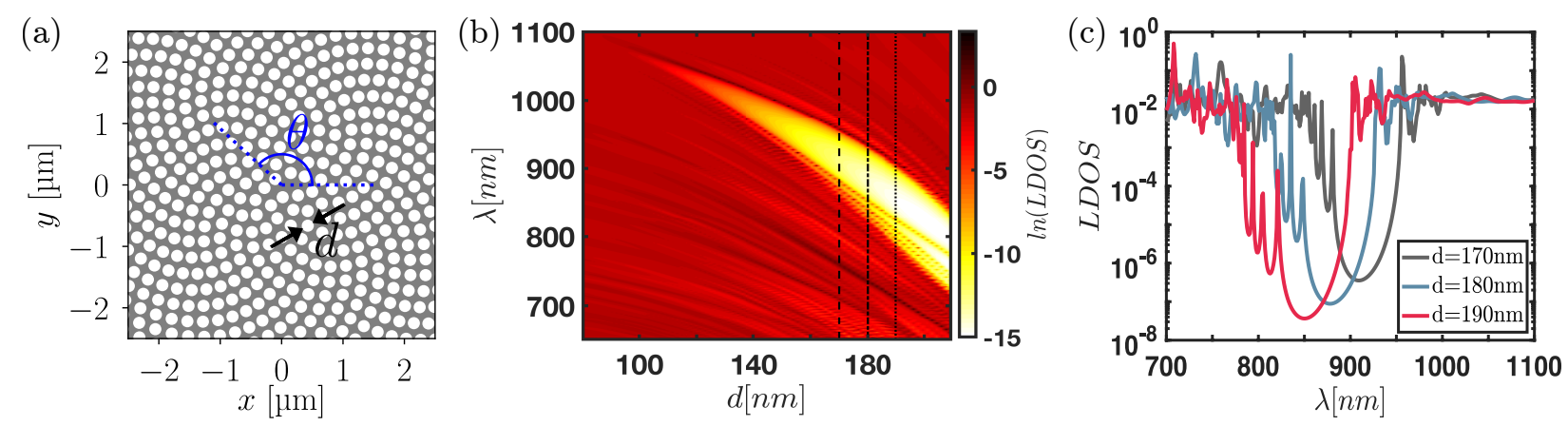

FIG. 1: GaAs golden-angle Vogel-spiral device designs. (a) Schematic of an example of an aperiodic photonic device under study with the relevant dimensions highlighted. The white circles represent air holes in the gray gallium arsenide membrane. (b) Local density of optical states (LDOS) map calculated at the center of the spiral as a function of the wavelength $\lambda$ and of the air-hole diameters $d$. (c) One dimensionalLDOS line-cut as a function of wavelength for three different air hole diameters: 170 (dark pastel grey line), 180 (dark pastel blue line), and $190 \mathrm{~nm}$ (dark pastel red line), for devices with an average inter-particle separation of $220 \mathrm{~nm}$. 

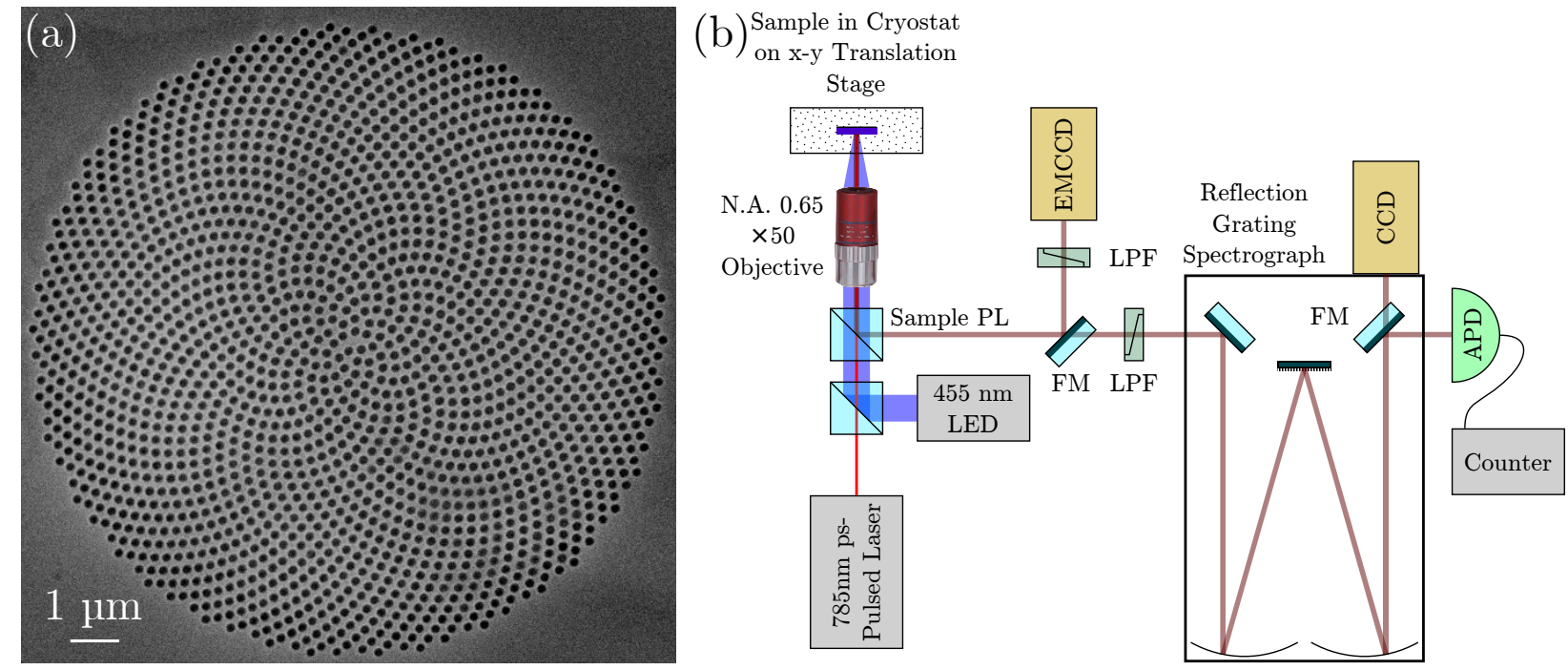

FIG. 2: Fabricated aperiodic nano-photonic devices and characterisation micro-photoluminescence setup. (a) Scanning electron micrograph of a suspended aperiodic photonic device with golden-angle Vogelgeometry. (b) Schematic of the confocal micro-photoluminescence set-up (not to scale), comprising a light emitting diode (LED) with emission wavelength centred at $455 \mathrm{~nm}$, a picosecond(ps)-pulsed $785 \mathrm{~nm}$ laser as excitation sources, focused by a $50 \times$ microscope objective (with numerical aperture NA $=0.65$ ) onto a sample placed on an $x y$-translation stage, within a cryostat for cryogenic measurements. The detection is carried out by an Electron Multiplying Charge Coupled Device (EMCCD) for photo-luminescence imaging, by a CCD for spectral characterisation and by an avalanche photo-diode (APD) with photon counting electronics, at the exit port of a reflection grating spectrometer, for time-resolved measurements. ( $\mathrm{LPF}=550$ and $800 \mathrm{~nm}$ long-pass filter, FM = flip mirror), the squares represent beam-splitters. 


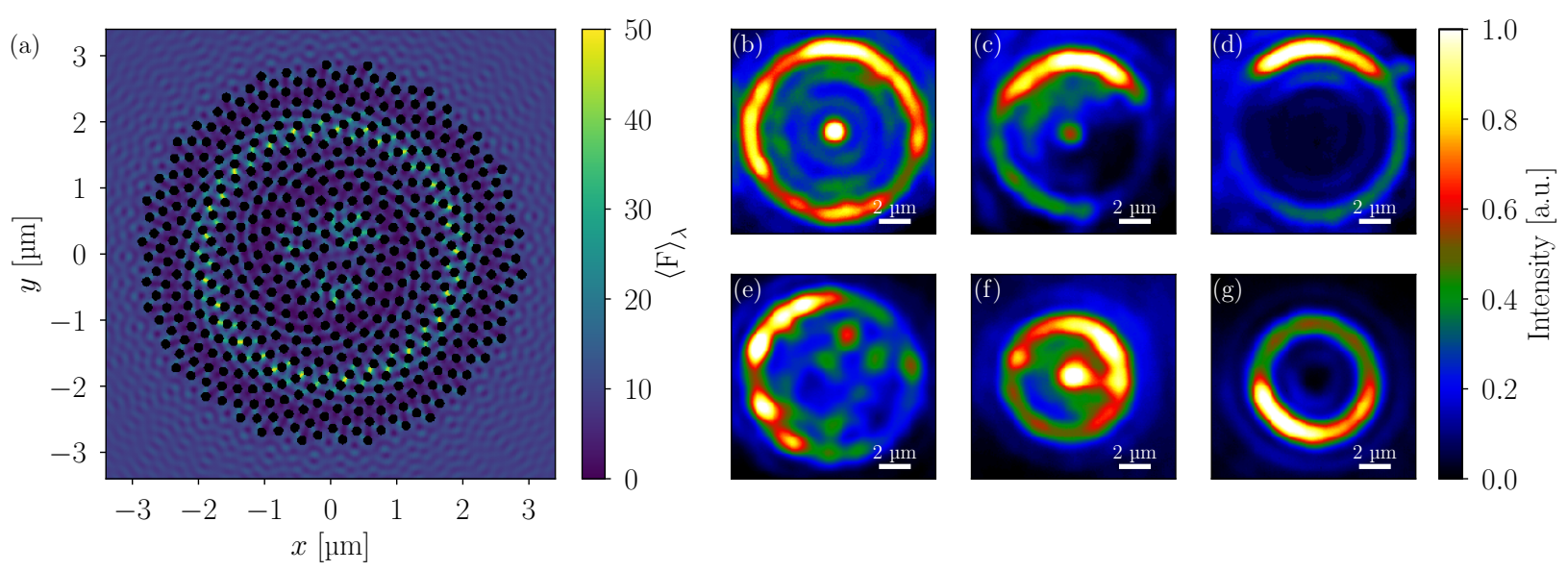

FIG. 3: Confined optical modes in aperiodic nano-photonic GaAs devices. (a) Simulation of the spectrally integrated LDOS $\left(\langle\mathrm{F}\rangle_{\lambda}\right)$ superimposed to the aperiodic device structure. (b-g) InAs quantum dots grown in the middle of the GaAs membranes are excited by a $455 \mathrm{~nm}$ LED with a power density of $40 \mathrm{~W} / \mathrm{cm}^{2}$, at a temperature of $10 \mathrm{~K}$, and the emitted light is collected by an EMCCD, after a $550 \mathrm{~nm}$ long-pass filter. Photo-luminescence images of the optical modes confined by the GaAs aperiodic nano-photonic devices are shown in panel (b) from a device with hole diameter $\mathrm{d}=190 \mathrm{~nm}$ and average centre-to-centre spacing $\mathrm{p}=240 \mathrm{~nm}$, panel (c) from a device with $\mathrm{d}=160 \mathrm{~nm}$ and $\mathrm{p}=230 \mathrm{~nm}$, panel (d) from a device with $\mathrm{d}=160 \mathrm{~nm}$ and $p=240 \mathrm{~nm}$, panel (e) from a device with $\mathrm{d}=180 \mathrm{~nm}$ and $\mathrm{p}=230 \mathrm{~nm}$, panel (f) from a device with $\mathrm{d}=180 \mathrm{~nm}$ and $\mathrm{p}=240 \mathrm{~nm}$, panel $(\mathrm{g})$ from a device with $\mathrm{d}=190 \mathrm{~nm}$ and $\mathrm{p}=230 \mathrm{~nm}$. 

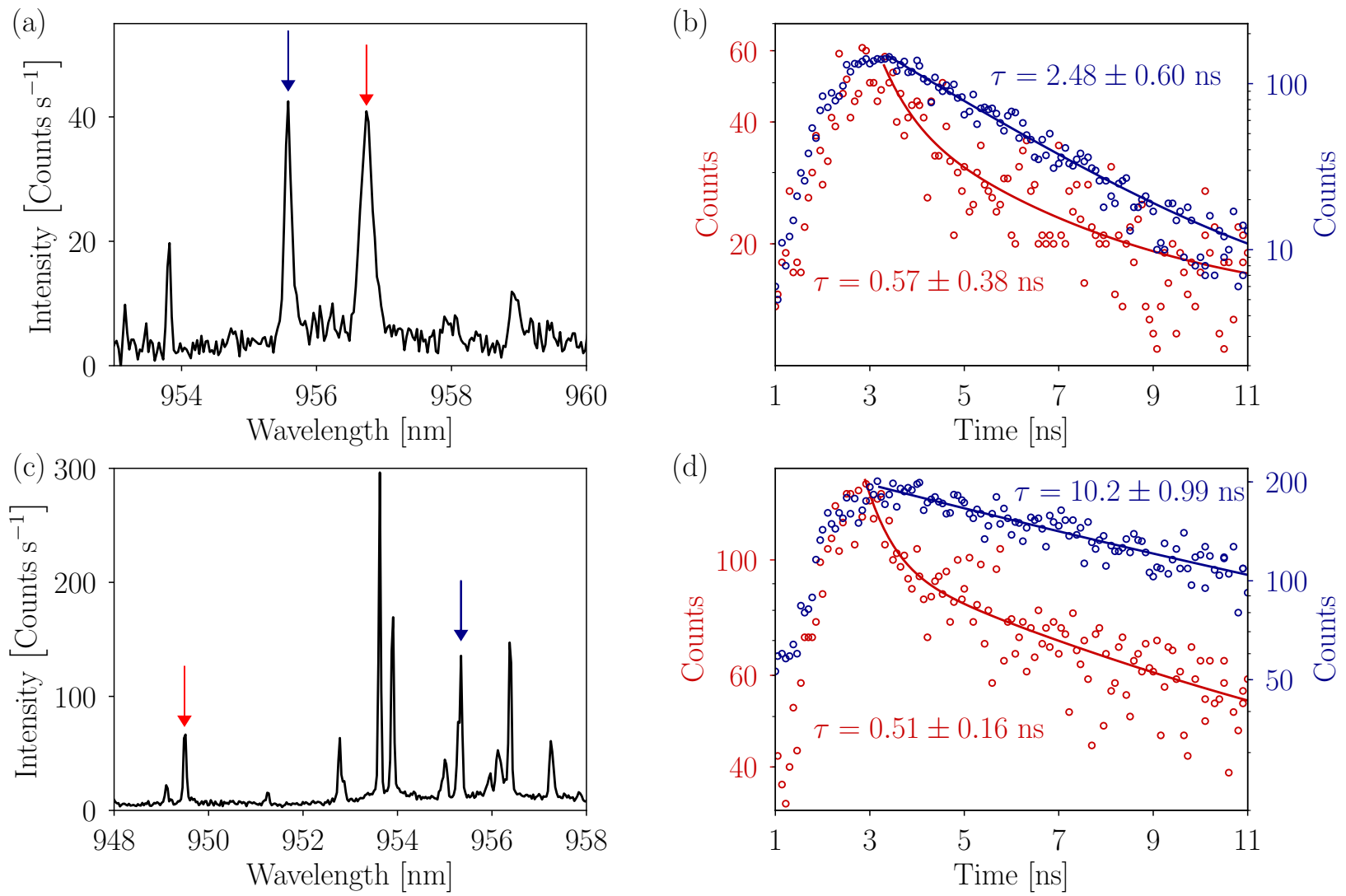

FIG. 4: Cavity quantum electrodynamics with InAs/GaAs quantum dots embedded within aperiodic nanophotonic devices. (a,c) Photo-luminescence spectra collected on a CCD under $780 \mathrm{~nm}$ laser illumination with power density of $18 \mathrm{~W} / \mathrm{cm}^{2}$ (panels (a,b)) and $339 \mathrm{~W} / \mathrm{cm}^{2}$ (panels $(\mathrm{c}, \mathrm{d})$ ), at a temperature of $10 \mathrm{~K}$. (b,d) Time-resolved measurement of the intensity of the light emitted by the optical transitions highlighted (and colour-coded) by the arrows in panels (a,c), collected by an avalanche photo-diode. Solid lines are exponential fits to the data (symbols) and the extracted decay time characteristics (lifetimes $\tau$ ) are shown. 